\title{
TEN YEARS OF PREOPERATIVE AUTOLOGOUS BLOOD DONA- TION IN ACCRA
}

\author{
${ }^{*}$ JUSTINA .K. ANSAH and J.K. ACQUAYE ${ }^{1}$ \\ National Blood Transfusion Service, P.O BOX KB 78 Korle Bu, Accra, Ghana and ${ }^{1}$ Department of \\ Haematology, University of Ghana Medical School, Accra, Ghana
}

\begin{abstract}
SUMMARY
Background - Preoperative autologous blood donation (PABD) is utilized to circumvent the use of allogenic blood for various reasons.

Objective - To describe the distribution in terms of demographic characteristic, trends in participation and result of screening test of the PABD programme of the Accra Area Blood Center from 1993-2003.

Design and Setting Retrospective descriptive study of PABD in patients scheduled for a variety of elective surgical procedures.

Setting- Different levels of institutional health care in Accra, Ghana.

Methods Data from existing records of patients who had participated in PABD were collated and analyzed

Results Five hundred and forty six (546) females and 89 males participated, with ages ranging between 14-74 years. Majority of the patients (76.7\%) underwent gynaecological surgery. A total of $330(52 \%)$ donated one unit only, and 299 $(47.1 \%)$ donated two units. Majority of the patients $(56.4 \%)$ had the surgery at the Korle-Bu Teaching Hospital (KBTH). Of the donations, 21 $(3.3 \%), 1(0.2 \%), 1(0.3 \%)$ and nil were positive for $\mathrm{HBV}$, HIV I \& II, HCV and VDRL respectively. A total of $848(89.4 \%)$ autologous crossmatched units were issued out. There was a steady progressive increase in participation.

Conclusion Mainly adult females scheduled for gynaecological surgeries in KBTH participated, while almost equal proportions donated one or two units of blood which meets the blood needs of most elective surgeries. Therefore healthy patients going for elective surgeries in regions with limited blood supply must be encouraged to enter a PABD Programme. Further studies in this field should evaluate motivational factors for participation
\end{abstract}

Keywords: Autologous donation, Gynaecological surgery, Accra, Blood transfusion

\footnotetext{
* Author for correspondence
}

\section{INTRODUCTION}

Autologous blood transfusion (ABT) is defined by the council of Europe as a transfusion in which the donor and recipient is the same person, who will be transfused with pre-deposited blood or its components ${ }^{1}$. The forms of ABT currently available are Preoperative Autologous Blood Donation (PABD), Acute Normovolemic Haemodilution (ANH) and Blood Salvage (BS) as intra-operative or postoperative procedures. Autologous blood can be used alone or in combination with allogenic blood. ABT is considered where blood loss requiring transfusion has occurred or is anticipated. In emergency, it may be the only readily available source of blood, as in the management of ruptured ectopic pregnancy in deprived district hospitals.

PABD involves the collection and storage of patient's blood prior to a planned surgery. This option is an alternative to allogeneic blood for patients whose general condition fall within donor guidelines and whose intraoperative blood requirements can be reasonably and accurately assessed preoperatively. A unit of the patient's blood is collected at intervals of seven days in the period leading to surgery. The blood is tested, labelled and stored under the same conditions as allogeneic blood. Autologous blood is made available on the day of surgery for transfusion to the patient. PABD may be utilized for a variety of planned surgical procedures. Before the advent of HIV/AIDS, a number of reasons were advanced in support of ABT. These included reduction of the need for recruited blood donors, reduction of the risk of transmission of hepatitis and reduction of the incidence of recipient alloimmunisation ${ }^{2}$. In the early years of the HIV scare, ABT was advocated as a means of reducing HIV transmission but later, this lost some of its impact because of higher cost and the improvement in safety procedures in blood collection, testing and transfusion. PABD had been used as a source of blood supply for patients with antibodies to high incidence blood group antigens 
and also for patients with rare blood groups ${ }^{2}$. The use of BS is rapidly increasing due to improved methods of collection, washing and reinfusion. BS using crude methods had been in use since $1925^{2}$. Shed blood in closed, sterile serosal cavity was collected, filtered through layers of sterile gauze and reinfused ${ }^{2,3}$. The need for use of PABD in Accra arose when many healthy patients coming for gynaecological surgery could not get blood pre deposited on their behalf. They resorted to either asking for favours from the blood centre, thereby putting pressure on limited stock levels or hiring commercial donors for a fee which compromised the safety of the blood stock. A decision was taken to educate healthy females scheduled for myomectomy or hysterectomy to donate blood for themselves. Patients for other planned surgeries were later encouraged to join the programme.

The Accra Area Blood Centre (AABC) sited at Korle Bu Teaching hospital (KBTH) is responsible for the blood transfusion needs of all patients in hospitals in Greater Accra and parts of Central and Eastern regions of Ghana. The objective of this study was to analyse the PABD programme over the period it had been operated to determine its scope, usefulness and impact to fashion out its future direction.

\section{MATERIALS AND METHODS Donors/Patients}

From February 1993 to December 2003, all healthy patients for myomectomy or hysterectomy calling at the AABC to solicit for assistance for blood donors for their planned surgery were recruited into a PABD programme. In the course of time healthy patients for other planned surgeries were also recruited into the programme. Patients were first given an educational talk on their health, the advantages and disadvantages of PABD and the requirements to enter the programme. The same criteria for selection of voluntary blood donors were used except that a prospective donor/patient must have an initial and subsequent haemoglobin $(\mathrm{Hb})$ level of $\geq 11 \mathrm{gm} / \mathrm{dl}$. No strict weight requirements were applied. Suitable adjustment to $10 \%$ total blood volume and anticoagulant quantity were made for donors with a weight of less than $50 \mathrm{kgs}$ (Immediately prior to venepuncture, some of the anticoagulant in the bag is allowed to drain away into a container by gravity, with care being taken not to allow air to enter the bag). No strict age limit was applied. Prospective donors/patients with ages greater than 70yrs or less than 16yrs were accepted after a careful clini- cal assessment and consultation with their attending physician.

Controlled mild to moderate hypertensives on medication, diabetics on oral hypoglycaemic agents and asthmatics in remission were also accepted. In addition, there must be sufficient time before surgery to enable the patient to donate the number of units required. The date for the surgery should be fixed and in the case of minors they must be willing, cooperative and parental consent given.

\section{Blood Collection}

A PABD request form which is a short communication between the doctor at the centre and the attending surgeon was completed after the patient's surgeon, anaesthetist, or the doctor at the blood center had educated the patient on the option of autologous transfusion. Informed consent of the patient and parents in the case of minors was obtained. The completed form was then sent to the blood center. Prospective donors/patients were clinically assessed by a doctor at the blood centre for suitability to enter the programme. Blood collections were scheduled at weekly intervals, with a week between the last collection and the date for the surgery. All donors/ patient took oral iron supplements as ferrous sulphate $65 \mathrm{mg}$ elemental iron three times daily for 2-4weeks, starting 1-2 weeks before the procedure. The $\mathrm{Hb}$ was checked before each collection and the patient was deferred if it was below $11 \mathrm{gm} / \mathrm{dl}$.

Patients with acceptable $\mathrm{Hb}$ levels then went through pre-donation counseling, medical assessment and a clinical form was completed. Education of patients who enrolled in the programme was paramount to the success of the procedure. This included advantages of the option of autologous transfusion and the steps involved in blood donation. Pre-donation counseling covered all aspects of microbiological screening tests to be performed on the donated blood as well as grouping and cross-matching with patient sample on the day of issue. Most patients that enrolled in this programme wanted reassurance that they would receive their own blood and not homologous blood. The possibilities of the results that might be obtained were discussed. The results were also discussed during post-donation counseling. Patients whose blood tested positive for Hepatitis B and $\mathrm{C}$ antibodies had the option of being transfused with their own blood or with homologous blood. In the blood collection procedure, each blood bag was carefully labelled as 'autologous' 
and with a special batch number e.g. $\mathrm{JKA}_{1} / 0021 / 04$ signifying the donor's initials, number of donation for particular surgery, number in autologous register and year of donation. This was explained to the donor/patient adequately to reassure them before collection began. The procedure for blood collection was as for collection of allogeneic blood, with all blood collections done under the supervision of a doctor. A special slip (Figure 1) was then given to the patient when the collection(s) were completed. ABO, Rh 'D' grouping and all available microbiological screening tests were performed on donor blood samples. All donor blood was tested at the early stages of the programme for HIV I \& II antibodies and Hepatitis B surface antigen only. Later on in the programme, VDRL for syphilis was reintroduced and Hepatitis $\mathrm{C}$ virus (HCV) antibody testing was introduced. Corresponding blood bags were labelled with the results. Units confirmed reactive for $\mathrm{HBsAg}$, anti HCV or VDRL were carefully labelled as biohazard "restricted only to autologous patient's use" whilst units confirmed reactive for anti-HIV were discarded appropriately. Autologous blood was stored in a dedicated compartment in the blood storage refrigerator.

\section{Blood Transfusion Procedure}

A completed blood request form, together with autologous blood collection slip and $5 \mathrm{mls}$ of patient's blood sample were sent to the blood bank laboratory 48hours prior to the surgery. Compatibility testing was performed for each unit of autologous blood against patients' blood sample. Autologous blood was then issued out on the day of the surgery. Patient's surgeon completed a specifically designed form on the day of discharge of the patient. This was sent to the doctor at the blood center. Following confirmation from the surgeon that the patient would no longer require blood transfusion, all units of autologous blood labelled as biohazard were discarded whereas units negative for all microbiological markers were crossed over to the regular bank pool for homologous use.

\section{Analysis}

Records covering 10 years 10 months were compiled and analysed according to age, sex, hospital and type of surgery on a yearly basis.

\section{RESULTS}

A total of 672 patients were enrolled in the PABD Programme from February 1993 to December 2003. Of the total number, 37 patients $(5.5 \%)$ were excluded because they failed the clinical screening.
The main reason for failure was low haemoglobin level as a result of menorrhagia.

The remaining 635 patients comprised 89 males and 546 females. They were aged between 14-74 years, mean of 41.7 years, with a male: female ratio of 1:6.1. The age distribution is depicted in Figure 2.

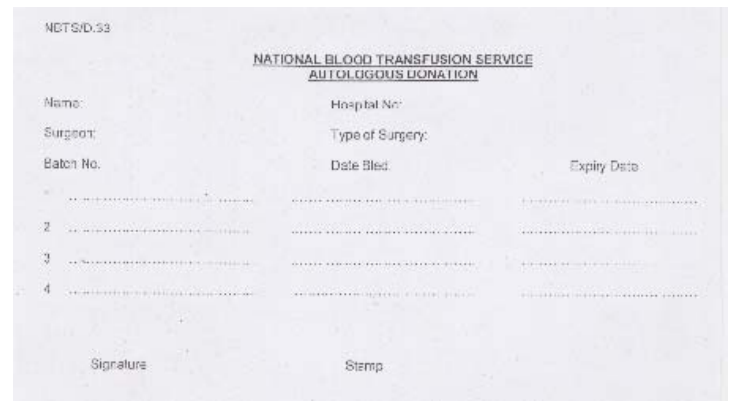

Figure 1 Autologous blood collection form

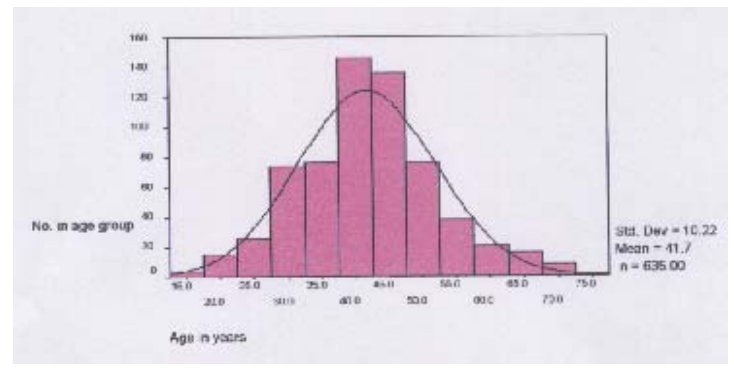

Figure 2 Age distribution of donors / patients who enrolled in the Preoperative Autologous Blood Donations programme.

Table 1 shows the distribution of patients undergoing PABD for various types of planned surgery.

Table 1 Type of surgery against number of patients

\begin{tabular}{lcc}
\hline Type of Surgery & Number & Percent \\
\hline Gynaecological Surgery & 487 & 76.7 \\
Neurosurgery & 52 & 8.2 \\
Orthopaedic Surgery & 37 & 5.8 \\
General Surgery & 21 & 3.3 \\
Maxillo-facial Surgery & 20 & 3.1 \\
Genitourinary Surgery & 13 & 2.0 \\
Plastic Surgery & 4 & 0.6 \\
Eye Surgery & 1 & 0.2 \\
\hline & & \\
Total & $\mathbf{6 3 5}$ & $\mathbf{1 0 0 . 0}$
\end{tabular}

Patients undergoing gynaecological procedures were the largest subgroup, representing $76.7 \%$ of 
the participants. Gynaecological procedures performed were myomectomy or hysterectomy for uterine fibroids, post menopausal bleeding, uterine prolapse, cervical lesions, removal of ovarian tumours, blocked fallopian tubes and repair of vesico-vaginal fistulas (VVF).

Neurosurgical procedures were mainly for cervical kyphoscoliosis, various forms of spinal stenosis, congenital scolosis/kyphosis and orthopaedic procedures were for spondylolisthesis and osteoarthritis (hip and or knee).

General surgical procedures were for removal of goiters or gallbladder, and repair of anal fistula, whilst maxillo-facial procedures were performed for mandibular, maxillary and parotid tumour resections. Genitourinary surgery performed included prostatectomy and removal of bladder or kidney mass. Plastic surgery procedures included correction for breast hypertrophy and excision of various tumours. The only eye surgery procedure was an orbitomy.

A total $330(52 \%)$ patients donated one unit of blood, $299(47.1 \%)$ donated two units and 6 $(0.9 \%)$ donated three units. The single unit donations were analysed to find out the category of surgery for which donations were made. The frequency of the one unit donations were as follows: Gynaecological surgery $281(85.2 \%)$, neurosurgery $14(4.2 \%)$, orthopaedic surgery $13(3.9 \%)$, genitourinary surgery $5(1.5 \%)$, faciomaxillary surgery $4(1.2 \%)$, the rest being plastic and eye surgery as $2(0.6 \%)$ and $1(0.3 \%)$ respectively. The yearly enrolment of patients who participated in the PABD programme from February 1993 to December 2003 is shown in Figure 3.

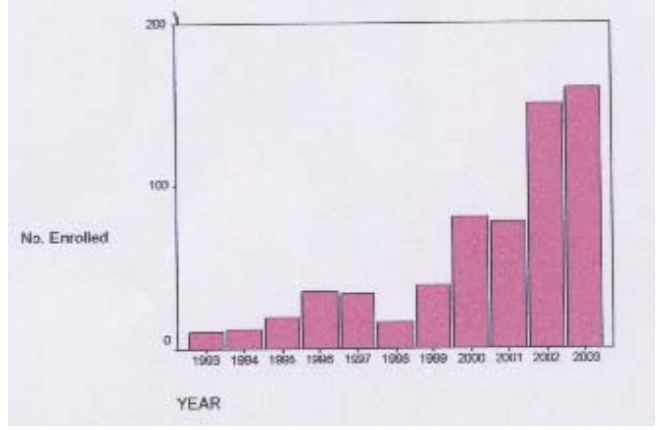

Figure 3 Yearly enrolment of patients who participated in the programme from February 1993 to December 2003.

There is a gradual rise in the number who entered the programme. There was no doctor at the centre from late 1997 to the first half of 1999 hence the fall during the period.

Patients who enrolled in the programme increased from 10 in 1993 to 166 in 2003 . Patients who participated in the programme were initially from the Korle $\mathrm{Bu}$ Teaching Hospital (KBTH). In the course of the observation period, after some modest education on the option of autologous transfusion, patients from other hospitals in the catchment area joined in the programme. Table 2 shows the hospitals from which patients originated. One patient reported from Battor Hospital in the Volta region.

Table 2 Hospitals from which patients were drawn

\begin{tabular}{|c|c|c|}
\hline Hospital & $\begin{array}{c}\text { No. of } \\
\text { Patients }\end{array}$ & $\begin{array}{l}\text { \% of } \\
\text { Total }\end{array}$ \\
\hline KBTH & 358 & 56. 4 \\
\hline Private Hospitals (Accra and & & \\
\hline Tema) & 240 & 37.8 \\
\hline Quasi Govt. Hospitals & 13 & 2.0 \\
\hline Other Govt. Hospitals (Accra) & 23 & 3.6 \\
\hline Hospitals outside Accra & 1 & 0.2 \\
\hline $\begin{array}{l}\text { Total } \\
\text { KBTH refers to Korle Bu Teaching Hospital. }\end{array}$ & 635 & 100 \\
\hline
\end{tabular}

The microbiological screening results of donated blood for the routine microbiological markers HIV I \& II, HBV, HCV, VDRL were as follows; positive HBV $21(3.3 \%)$, positive HCV $1(0.3 \%)$, positive VDRL $0(0 \%)$, positive HIV I \& II $1(0.2 \%)$. A total of 848 units of compatible blood (89.4\%) were issued out during the period after crossmatching from the blood centre laboratory to respective hospitals and clinics for patient transfusion.

\section{DISCUSSION}

The importance of autologous blood transfusion had been increasingly recognized in the last 25 years $^{4}$. The percentage of autologous blood transfused yearly in USA rose from $0.8 \%$ in 1982 to $8.1 \%$ in $1992^{5}$. In Italy and Germany the yearly percentages of autologous blood transfused in 2000 were $7.8 \%$ and $6.4 \%$ respectively ${ }^{1}$. PABD had been used as a tool for conservation of bank blood for cardiopulmonary and orthopaedic operations. Its introduction at the Accra Area Blood Centre for Korle $\mathrm{Bu}$ Teaching Hospital patients was mainly for blood conservation. Seventy percent $(70 \%)$ of blood donations to the Accra Area Blood Centre (AABC) were pre-deposit family 
donations for planned surgery or replacement donations. Patients' relatives deposited blood before patients were booked for surgery. In over $30 \%$ of patients, the blood was not utilized. This non utilization was carried over into the PABD.

The main goal of a preoperative autologous blood donation programme in most centers is to reduce or eliminate the need for homologous blood transfusion. While PABD represents good transfusion practice for surgical procedures likely to require blood transfusion, its application in surgical procedures unlikely to require blood transfusion is controversial $^{6,7,8}$. Potential candidates for PABD prior to surgery include any patient scheduled for a procedure for whom cross-matched blood is requested, indicating a likelihood of requiring blood transfusion according to a maximum surgical blood ordering schedule. It had been observed that in over $60 \%$ of gynaecological surgery, collected autologous blood was not used ${ }^{9}$. Of those who received transfusion, $61 \%$ were given one unit only ${ }^{9}$. In that study it was observed that if the probability of a patient receiving a transfusion with the surgery is less than 5\% there was no need for a PABD. This was common with gynaecological and plastic surgery. Fifty-two percent (52\%) of the patients in this study donated one unit towards their surgeries. In $74.1 \%$ of the cases, surgeons requested single unit donation whilst the rest had $\mathrm{Hb}$ levels below the accepted level of $11.0 \mathrm{gm} / \mathrm{dl}$ at subsequent donations and were therefore excluded from further donation. Two patients who participated in the PABD programme also had Acute Normovolemic Haemodilution (ANH) in theatre on the day of surgery. ANH is essentially an anaesthetic procedure that involves the removal of patient's red blood cells by controlled removal of whole blood and the simultaneous replacement with a combination of colloid or crystalloid to maintain adequate circulatory volume. This procedure is usually performed in the operating room in the perianaesthesia period with standard or invasive monitoring in place. The advantage of $\mathrm{ANH}$ in addition to providing autologous blood is that it brings about improved blood flow distribution within the microvascular network and enhanced tissue perfusion ${ }^{10}$. These single unit donations were mainly for gynaecological surgery known for low likelihood of transfusion but which in the KBTH setting blood had to be pre-deposited. In this programme autologous blood donation increased from $10(0.08 \%$ of total donations $)$ in 1993 to 160 ( $0.9 \%$ of total donations) in 2003 . Gynaecological surgery is by far the largest category constituting $76.7 \%$. Despite this increase the number is very small, being less than $1 \%$ of the total blood donations for that year (NBTS annual report). Over the years, there was a gradual expansion of the programme to cover other hospitals and also for non gynaecological surgery. About thirty-seven percent $(37.8 \%)$ of the patients were from private hospitals. This is significant, considering that between $8 \%$ to $17 \%$ of total blood collections for 2002-2003 were issued to private hospitals compared to $37.8 \%$ of autologous. The frequency of microbiological markers was not remarkably lower than in the voluntary donor populations except for HIV which was low at $0.3 \%$.

The advantages of PABD are enormous. These are that patients benefit from knowing that they were actively involved in their own treatment and the phlebotomy process also stimulates the bone marrow to increase cell production. This stimulated blood production is thought to speed up a patient's recovery postoperatively ${ }^{11}$. PABD had been shown to result in accelerated peri-operative erythropoeisis $^{12}$. The patient's privacy with respect to the surgery was assured since the blood needs towards the particular surgery were donated by him/her. PABD has the advantage of ensuring reduced pressure on bank blood which can then be used for the blood needs of medical and surgical emergencies. PABD as a method of blood transfusion presents no risk of transmitted viral infection or development of alloantibodies, avoids the alleged problem of increased postoperative infections and enhanced risks of tumour recurrence ${ }^{13}$, and is an outlet for people with rare or unusual blood group.

PABD however requires considerable planning and organization. The criteria for patient eligibility are well defined and some enthusiastic patients will just not be fit enough to be included in the programme. One major disadvantage realized in this study is inappropriate usage which results in wastage of blood. Surgeons should not transfuse autologous blood simply because it is available. This wastage was also observed by others ${ }^{9}$. Another drawback was the inability of clinicians to provide desired information on the fate of the blood collected. An autologous blood transfusion form specifically designed for the purpose to indicate the outcome of blood was not completed in most instances. The cost of autologous blood transfusion has been a drawback in many centers but this is because in these centers the unused blood is discarded ${ }^{5}$. This is not an issue in this centre where those unused units which are negative with microbiological screening are added to the bank pool. 
Iron replacement alone or together with marrow stimulation with erythropoietin is required. The latter is required when aggressive PABD is anticipated, by which $1000 \mathrm{mls}$ blood is removed a week. Erythropoietic response to serial phlebotomy over an interval may result in iron-restricted erythropoiesis, endogenous erythropoietic response or both ${ }^{14,15}$

Procedural and clerical errors must be consciously avoided and therefore the same conditions observed for allogeneic donations are ensured for autologous blood donation. Bacterial contamination is a remote risk just as allogeneic blood. Similar conditions are observed during blood collection, storage and processing of autologous blood.

\section{CONCLUSION AND RECOMMEN- DATION}

It is observed that preoperative autologous blood donation had increased over the ten year period. It is, however, less than 1 percent of blood collected. It is a solution for providing blood needs of many healthy patients requiring planned surgery. Surgeons and Gynaecologists working in teaching hospitals and regional hospitals are encouraged to educate these healthy patients to opt for PABD.

\section{ACKNOWLEDGEMENT}

The authors wish to thank Dr. Lucy Asamoah Akuoko, Deputy Director of the Accra Area Centre for her assistance in the programme.

\section{REFERENCES}

1. Politis C, Richardson SC. An update of predeposit autologous blood donation and transfusion in Europe: Vox Sang 2004; 87: 105-108.

2. Swisher SN, Petz LD. Autologous blood transfusion and blood salvage in clinical practice of blood transfusion by Petz L.D., Swisher S. N., Churchhill Livingstone, New York $1^{\text {st }}$ edition $1981 ; 345-356$.

3. Ekem I, Obed SA. Intra operative blood salvage in comprehensive obstetrics in the tropics by Kwawukume E.Y., Emuveyan E.E; Asante and Hittscher Printing, Accra. $1^{\text {st }}$ edition 2002; 300-301.

4. Goodnough LT, Shafron D, Marcus RE. The impact of preoperative autologous blood donation on orthopaedic surgical practice. Vox Sang 1990; 59: 65-69
5. Goodnough LT, Monk TG, Brecher ME. Autologous blood procurement in the surgical setting; Lessons learned in the last 10 years. Vox Sang 1996; 71: 133-141

6. Aubuchon JP. Autologous transfusion and directed donations. Current controversies and future directions. Transfus Med Rev 1989; 3: 290-306

7. Chambers C, Kruskall MS. Preoperative autologous blood donation. Transfus Med Rev 1990; 4 : 35-46

8. Sayers M. Autologous blood donation in pregnancy. Con Transfusion 1990; 30: 172174

9. Goodnough LT, Saha P, Hirschler NV and Yomtovian R. Autologous blood donation in non orthopaedic surgical procedures as a blood conservation strategy. Vox Sang 1992; 63: 96-101

10. Aryeh S, Tanuja SR. Acute normovolemic hemodilution. Transfus 2004; 44(Suppl): 26s$34 \mathrm{~s}$.

11. Wright PA. Autologous donor selection in modern blood banking by Harmening D.M; F.A. Davis Company. Philadelphia. $3^{\text {rd }}$ edition 1994; 207-203

12. Levine E, Rosen A, Seghal L, Gould S, Seghal $\mathrm{H}$, and Moss G. Accelerated erythropoesis: The hidden benefit of autologous donation. Transfus 1990; 30: 295-297.

13. Napier JAF. Autologous transfusion and blood conservation in hand book of blood transfusion therapy by Napier J.A.F: John Wiley and Sons. Chichester. $2^{\text {nd }}$ edition 1996; 214-225

14. Goodnough LT and Brittenhem GM. Limitations of the erythropoeitic response to serial phlebotomy. Implications for homologous blood programs. J Lab Clin Med 1980; 115: 28-35

15. Goodnough LT, Price TH and Rudnick S. Iron restricted erythropoeisis in the erythropoietic stimulated bone marrow. J Lab Clin Med 1991; 118: 289-296 